\title{
Buried myoepithelial stem cells as a reservoir for repairing the exposed airway epithelium
}

\author{
Carlotta Duval $^{1,2}$, Mika Watanabe ${ }^{1,2}$, Giacomo Donati $^{1,2}$ \\ ${ }^{1}$ Department of Life Sciences and Systems Biology, ${ }^{2}$ Molecular Biotechnology Center, University of Turin, Torino, Italy \\ Correspondence to: Giacomo Donati. Department of Life Sciences and Systems Biology, University of Turin, Via Accademia Albertina 13, 10123, \\ Torino, Italy. Email: giacomo.donati@unito.it. \\ Comment on: Tata A, Kobayashi Y, Chow RD, et al. Myoepithelial Cells of Submucosal Glands Can Function as Reserve Stem Cells to Regenerate \\ Airways after Injury. Cell Stem Cell 2018;22:668-683.e6.
}

Received: 11 September 2018; Accepted: 09 November 2018; Published: 06 December 2018.

doi: 10.21037/sci.2018.11.07

View this article at: http://dx.doi.org/10.21037/sci.2018.11.07

The tracheal epithelium that connects the upper airway to the lung consists of two compartments, the submucosal gland (SMG) and the surface airway epithelium (SAE). Less exposed to environmental hazards, myoepithelial cells (MECs) in SMG represent a stem cell reservoir to repair severely injured surface epithelia $(1,2)$.

Epithelial tissues are composed of tightly assembled cells that cover a surface or line a cavity. Some epithelia consist of a single cell layer (i.e., in the respiratory tract and intestine), while others are stratified (i.e., in skin and esophagus). One interesting feature of some epithelia is the presence of adnexal structures often associated with glandular structures with dedicated functions, such as the pilosebaceous units in the skin and SMG in airway epithelium $(3,4)$. Epithelia exposed to external environment have the crucial role to protect the underneath tissue acting as physical, chemical and immunological barrier. They can also be involved in absorptive and secretory processes through specialized individual cells or cells organized in glandular structures. Epithelia and their associated adnexal structures are maintained by stem cells always located on the basement membrane, able to self-renew and generate progeny that undergoes differentiation (5). Epithelia are characterized by a continuum of distinct and spatially separated epithelial lineages that are maintained by dedicated stem cells. This compartmentalization is strictly maintained in homeostasis and, if altered, can lead to pathological conditions (6). During tissue injury, many epithelial cell populations have been shown to exhibit a remarkable plasticity contributing to the repair not only of their own lineage, but also to distinct injured epithelial lineages $(4,7,8)$. In several epithelia, cells of the adnexal structures migrate out of their homeostatic niche to become the new resident progenitors of the repaired epithelial injury. This is the case of the epidermis, the outermost layer of skin, where the epidermal cells resident in the pilosebaceous unit or in the sweat gland can contribute to the interfollicular and paw epidermis regeneration respectively (9-11). In some cases, it has been observed that a small proportion of these cells will remain resident for a relative long term even after the complete resolution of the injury $(9,10)$. In the epidermis this change in location within the epithelium has been shown to be associated with a lineage reprogramming that is initially characterized by a loss of the original identity of the injury-engaged stem cells, followed by a transient lineage infidelity, and later by the acquisition of the lineage identity of the repaired epithelial niche $(10,12)$. Together with the stem cells, also some differentiated cells contribute to the healing process. Recent studies have shown that in epidermis, in the lung and in the intestine a tissue lesion can stimulate differentiated or committed cells to revert back to a more stem-cell state $(10,13)$. For example, lineage tracing in vivo experiments showed that the luminal secretory cells of the airway can dedifferentiate into stem cells, functioning as their endogenous counterparts in repairing epithelial injury (14).

Together with lineage tracing technology, that has revealed all the previously mentioned cell behaviors in vivo, single cell (sc) expression profiling represents a powerful discovery method to deeply understand tissue heterogeneity. Even in case of rare subpopulations, scRNA-Seq is able to 
identify cell types or cell states and to link this knowledge to positional information (15-17). The computational analysis of scRNA-Seq offers the possibility to predict, from a single snapshot, the cellular transitional and dynamic states. For example, in the lung epithelium scRNA-seq has revealed the lineage hierarchies in homeostasis (18).

Located below the SAE of cartilaginous airways, SMGs are tubuloacinar structures that secrete antimicrobial mucous and serous fluids fundamental for the innate immunity in the airway epithelium. They consist of ciliated and collecting ducts, mucous tubules and serous acini. The ducts that connect the acini to the SAE are composed of cells similar to the SAE cell types: stem and luminal cells (secretory, ciliate and club cells). Conversely, tubules and acini, the more distal structures from the SAE, are characterized by unique cell types: the serous and mucous luminal cells and the contractile MECs which are thin cells, with a spindle or stellar-shape, situated between the basal membrane and the other epithelial cells $(1-3,19)$. In homeostatic condition, since SAE and SMG stem cells are mostly quiescent, the tracheal epithelial turnover is very slow. However, after injury, they proliferate to repair their own compartment. In an in vivo model of tracheal epithelial regeneration using syngeneic subcutaneous tracheal transplantation, it has been shown that SMG duct cells can reconstitute functional SMG-like structures (20). In addition, lineage tracing of KRT14+ SMG duct cells showed their ability to contribute to the SAE repair following a severe injury (20).

Recently, the combination of scRNA-Seq and lineage tracing experiments revealed the plasticity of MECs resident in the SMG of the airway epithelium after injury $(1,2)$.

Through scRNA-Seq, Tata et al. identified a cluster of cells in the SAE expressing SMG markers upon naphthalene-mediated injury, that induces sloughing and death of SAE cells. Clustering of the expression profiles revealed co-expression of genes related to normal airway luminal and stem cells, as well as markers of developing SMGs $\left(\mathrm{SOX}^{+}\right)$or MEC (ACTA2 ${ }^{+}$, also known as $\alpha$-SMA alpha smooth muscle actin). The histological analysis during the SAE healing dynamics identified highly proliferative and migratory cells in the SAE expressing SMG markers. Different injury airway models, such as sulfite dioxide administration or influenza virus infection, led to a lower degree of damage and to a fewer ACTA2 ${ }^{+}$and $\mathrm{SOX}^{+}$ SMG-like cells suggesting that the SAE repair contribution by SMG cells is linked to injury severity.

To prove the SMG cells plasticity during SAE injury, lineage tracing of SOX9 expressing cells was performed. After injury, a large percentage of SOX9-labeled cells derived from SMG were observed not only in the proximal, but also in the distal SAE. These cells were characterized by the expression of stem, secretory or ciliated markers, indicating a broad cellular contribution of SMG in the SAE repair. Interestingly, the number of secretory and ciliated cells derived from SMG SOX ${ }^{+}$cells increased overtime, suggesting a model in which SMG cells migrated in the injured SAE would differentiate into specialized cells to establish a proper airway barrier. To understand if the contribution of the SMG to the SAE healing is recapitulated by MECs, Tata and collaborators performed similar lineage tracing experiment labelling $\mathrm{ACTA}^{+}$cells. Inducing a severe injury of the tracheal epithelium, they observed that the ACTA2 $2^{+}$lineage contributes to stem and luminal cells in both SAE and SMG.

To further test MEC plasticity, ACTA2 ${ }^{+}$cells were isolated and cultured. In these conditions $\mathrm{ACTA} 2^{+}$cells stopped expressing ACTA2 and induced the expression of SAE stem cells markers and also changed their morphology, thus resembling SAE stem cells. Furthermore, in an ex vivo tracheal regeneration model in which SAE cells have been removed from a tracheal scaffold, ACTA2 ${ }^{+}$cells were able to proliferate and generate epithelial sheets composed of both stem and luminal SAE cells. All these data suggest that SMG-derived cells represent a stem/progenitor reservoir in mouse tracheal airways.

To test if this regenerative mechanism is also activated in large animals, they employed a porcine model of airway injury after exposure to chlorine gas. In this context only $\mathrm{SOX}^{+}$and not $\mathrm{ACTA}^{+}$cells were observed to be induced in the SAE. However, isolated porcine MECs are able to generate SAE stem-like cells in ex vivo cultures. The authors suggested that since SMGs are particularly abundant in porcine compared to murine airways, the SMG duct cells might alone be sufficient to repair injured SAE, a hypothesis that will need further analysis. To explore the molecular mechanism involved in MECs plasticity in mice, they analyzed the role of the transcription factor SOX9. Conditional genetic ablation of SOX9 in the tracheal epithelium led to a fewer number of MECs in injured SAE regions, with respect to control, and a concomitant reduction of proliferation. Therefore, similarly to other epithelial regenerative contexts, SOX9 represents an important player during the acquisition of MEC plasticity.

The plasticity of MECs upon injury shown by Tata $e t a l$. has been also identified and dissected by Lynch et al. Both 
studies defined MECs as a reserve of multipotent SAE and SMG stem cells. Indeed, Lynch et al. demonstrated through two lineage tracing models that, after severe airways injury, MECs migrate toward SAE where they proliferate and adopt a SAE stem cells phenotype. Like Tata et al., they showed that MECs resident in SAE are able to generate all SAE cell types: stem, secretory, ciliated cells and rarely club cells. This bias of SAE MEC-derived progenitors towards secretory and ciliated differentiation was partially rescued in a second injury where an increased number of club cells were observed. These data indicate that the differentiation potential of SAE MEC-derived progenitors and SAE stem cells is not equivalent (2).

Similar to the early stages of SMG morphogenesis, Lef-1, a Wnt-regulated transcription factor, is a key player of the MEC plasticity observed upon injury. Lynch et al. demonstrated that Lef-1 inducible over-expression improved tissue repair after naphthalene induced damage, increasing the number of replicating MECs in SMG and resident MECs in SAE. Moreover, they proved that Lef-1 over-expression in MECs is sufficient to drive transdifferentiation from MEC to stem SAE cells without tissue damage. This suggests that Lef-1 modulation in MEC-derived progenitors may be a strategy for regenerative medicine in lung diseases.

The recent reports from Tata $e t a l$. and Lynch et al., offer a new example of compartmentalized sheltered cells in an epithelial adnexal structure able to contribute to the repair of the more exposed area of the epithelium. This phenomenon has been observed for example in skin, where different epidermal cells restricted in homeostasis in the hair follicle, sebaceous gland and sweat gland, acquire the ability to migrate out their own epithelial niche and regenerate the epidermis during wound healing (7-11). All together these observations highlight the possibility of a general mechanism to keep specialized epithelial cells, holder of a remarkable plastic potential, in the adnexal structures of the epithelia, where they are protected, away from the regions of the epithelium more exposed to injury.

MECs are also located in other glandular epithelia, such sweat, mammary, lacrimal and salivary glands where contraction favors the gland secretion. These cells are epithelial cells, as their major components of intermediate filament system are Krt5 and Krt14, however they also have element of smooth muscle differentiation required for contraction. If the identity of MECs compared to other epithelial stem cell identities gives unique epithelial plasticity features is still an open question. It is interesting that in some contexts, such as in mammary glands, MECs show greater plasticity compared with their neighbor luminal long-lived progenitors in transplantation assay $(21,22)$.

While we are still learning the intrinsic potential of MECs during tissue repair, it is evident that plasticity triggering is dependent on distance from the injury and its extent. In sweat glands, for instance, ductal progenitors that are in closer proximity to the injury, but not glandular progenitors, participate to the epidermal wound repair (11). Similarly, the ductal cells in the SMG contribute to the repair even in minor SAE injuries $(1,2)$. On the same line, in large mammals where SMGs are more abundant and larger than in mice, even after important injury such as the one triggered by naphthalene, only the ductal cells, in proximity of the injured $\mathrm{SAE}$, contribute to the repair while the MECs do not (1).

In terms of molecular mechanisms, two crucial epithelial developmental players such as the transcription factors SOX9 and LEF1 have been shown to positively regulate SMG MEC plasticity during SE injury. Further studies will be necessary to better define how SOX9 targets are involved in the switch from SMG to SAE phenotype, and how LEF1 can influence both differentiation of SMG and dedifferentiation toward SAE phenotype. In addition, it will be important to identify key chromatin and epigenetic factors and verify if some negative regulators of plasticity, such as the transcription factor JUNB (a component of the AP-1 complex) in the epidermis (23) also exist in the SMG and SAE regenerative context.

\section{Acknowledgements}

We acknowledge Compagnia di San Paolo and Fondazione CRT for generous funding.

\section{Footnote}

Conflicts of Interest: The authors have no conflicts of interest to declare.

\section{References}

1. Tata A, Kobayashi Y, Chow RD, et al. Myoepithelial Cells of Submucosal Glands Can Function as Reserve Stem Cells to Regenerate Airways after Injury. Cell Stem Cell 2018;22:668-683.e6.

2. Lynch TJ, Anderson PJ, Rotti PG, et al. Submucosal Gland Myoepithelial Cells Are Reserve Stem Cells That 
Can Regenerate Mouse Tracheal Epithelium. Cell Stem Cell 2018;22:779.

3. Lynch TJ, Engelhardt JF. Progenitor cells in proximal airway epithelial development and regeneration. J Cell Biochem 2014;115:1637-45.

4. Donati G, Watt FM. Stem cell heterogeneity and plasticity in epithelia. Cell Stem Cell 2015;16:465-76.

5. Blanpain C, Horsley V, Fuchs E. Epithelial stem cells: turning over new leaves. Cell 2007;128:445-58.

6. Xian W, Ho KY, Crum CP, et al. Cellular origin of Barrett's esophagus: controversy and therapeutic implications. Gastroenterology 2012;142:1424-30.

7. Blanpain C, Fuchs E. Stem cell plasticity. Plasticity of epithelial stem cells in tissue regeneration. Science 2014;344:1242281.

8. Burclaff J, Mills JC. Plasticity of differentiated cells in wound repair and tumorigenesis, part II: skin and intestine. Dis Model Mech 2018;11(9). pii: dmm035071.

9. Page ME, Lombard P, $\mathrm{Ng}$ F, et al. The epidermis comprises autonomous compartments maintained by distinct stem cell populations. Cell Stem Cell 2013;13:471-82.

10. Donati G, Rognoni E, Hiratsuka T, et al. Wounding induces dedifferentiation of epidermal Gata6+ cells and acquisition of stem cell properties. Nat Cell Biol 2017;19:603-13.

11. Lu CP, Polak L, Rocha AS, et al. Identification of stem cell populations in sweat glands and ducts reveals roles in homeostasis and wound repair. Cell 2012;150:136-50.

12. Ge Y, Gomez NC, Adam RC, et al. Stem Cell Lineage Infidelity Drives Wound Repair and Cancer. Cell 2017;169:636-650.e14.

13. Tetteh PW, Basak O, Farin HF, et al. Replacement of Lost Lgr5-Positive Stem Cells through Plasticity of

doi: $10.21037 /$ sci.2018.11.07

Cite this article as: Duval C, Watanabe M, Donati G. Buried myoepithelial stem cells as a reservoir for repairing the exposed airway epithelium. Stem Cell Investig 2018;5:45.
Their Enterocyte-Lineage Daughters. Cell Stem Cell 2016;18:203-13.

14. Tata PR, Mou H, Pardo-Saganta A, et al. Dedifferentiation of committed epithelial cells into stem cells in vivo. Nature 2013; 503:218-23.

15. Proserpio V, Piccolo A, Haim-Vilmovsky L, et al. Singlecell analysis of CD4+ T-cell differentiation reveals three major cell states and progressive acceleration of proliferation. Genome Biol 2016;17:103.

16. Proserpio V, Lonnberg T. Single-cell technologies are revolutionizing the approach to rare cells. Immunol Cell Biol 2016;94:225-9.

17. Donati G. The niche in single-cell technologies. Immunol Cell Biol 2016;94:250-5.

18. Treutlein B, Brownfield DG, Wu AR, et al. Reconstructing lineage hierarchies of the distal lung epithelium using single-cell RNA-seq. Nature 2014;509:371-5.

19. Tata PR, Rajagopal J. Plasticity in the lung: making and breaking cell identity. Development 2017;144:755-66.

20. Hegab AE, Ha VL, Gilbert JL, et al. Novel stem/ progenitor cell population from murine tracheal submucosal gland ducts with multipotent regenerative potential. Stem Cells 2011;29:1283-93.

21. Van Keymeulen A, Rocha AS, Ousset M, et al. Distinct stem cells contribute to mammary gland development and maintenance. Nature 2011;479:189-93.

22. Prater MD, Petit V, Alasdair Russell I, et al. Mammary stem cells have myoepithelial cell properties. Nat Cell Biol 2014;16:942-50, 1-7.

23. Singh K, Camera E, Krug L, et al. JunB defines functional and structural integrity of the epidermo-pilosebaceous unit in the skin. Nat Commun 2018;9:3425. 